\title{
Über die Veresterung von Dioxyaceton mit Phosphaten.
}

\author{
Von \\ A. v. Lebedew. \\ (Aus dem agrikulturchemischen Laboratorium des Donauer Polytechnikums, \\ Nowotscherkask.) \\ (Der Redaktion zugegangen am 14. Februar 1913.)
}

Man hat mich aufmerksam gemacht auf die folgende Stelle in der Arbeit von Hans Euler und David Johanson: ${ }^{1}$ ) Versuche über die enzymatische Phosphatbildung,. Die Autoren schreiben folgendes: «v. Lebedew, welcher die.ersten Versuche (über die Veresterung von Dioxyaceton) angestellt hat, hatte ursprünglich angegeben, daß Dioxyaceton keine Phosphorsäureester bildet. Ohne Kenntnis dieses Versuches ist bald darauf der eine von uns (Euler) zum Ergebnis gekommen, daß eine, wenn auch geringe Esterbildung stattfindet. In einer bald darauf erschienenen Arbeit (Ber. d. Deutsch. Gesellsch., Bd. 44, S. 2932, 1911) findet auch Herr v. Lebedew, daß Dioxyaceton durch Phosphate verestert werden kann. ${ }^{2}$ )

Dazu möchte ich bemerken, daß ich meine Mitteilung über die Veresterung des Dioxyacetons bei der Gärung zuerst in den Comptes rendus de l'Acad. des Sciences ${ }^{3}$ ) veröffentlicht habe und später ausführlicher in den Berichten der Deutschen chemischen Gesellschaft. ${ }^{4}$ Die erste Mitteilung, die bedeutend früher erschienen ist, als die oben erwähnte Arbeit von $\left.\mathrm{Euler},{ }^{5}\right)$ ist gerade von Autoren nicht zitiert.

Man sieht also, daß ich meine Untersucbung ohne Kenntnis des Versuches von Herren v. Euler und Sixten Kullberg fortgesetzt habe.

1) Diese Zeitschrift, Bd. 80, S. 205 (1912).

2) 1. c. S. 206.

3) Compt. rend. de l'Acad. des Sciences, Bd. 153, S. 136 (1911).

4) 1. c.

j) Hans Euler und Sixten Kullberg, Diese Zeitschrift, Bd. 74, S. 15 (1911). 